\title{
基于机器学习的设备剩余寿命预测方法综述
}

\author{
裴 洪 胡昌华 司小胜 张建勋庞哲楠 张鹏 \\ (火箭军工程大学导弹工程学院 西安 710025)
}

\begin{abstract}
摘要: 随着科学技术的发展和生产工艺的进步, 当代设备日益朝着大型化、复杂化、自动化以及智能化方向发展。为保障设 备安全性与可靠性, 剩余寿命(Remaining useful life, RUL)预测技术受到了普遍关注, 同时得到了广泛应用。传统的统计数据 驱动方法受模型的选择影响明显, 而机器学习具有强大的数据处理能力, 并且无需确切的物理模型和专家先验知识, 因而机 器学习在剩余寿命预测领域表现出了广阔的应用前景。鉴于此, 详细分析和阐述了基于机器学习的设备剩余寿命预测方法。 根据机器学习模型结构的深度, 将其分为基于浅层机器学习的方法和基于深度学习的方法。同时疏理了每类方法的发展分支 与研究现状, 并且总结了相应的优势和缺点, 最后探讨了基于机器学习的剩余寿命预测方法的未来研究方向。
\end{abstract}

关键词: 剩余寿命预测; 机器学习; 神经网络; 支持向量机；深度学习

中图分类号: V448

\section{Review of Machine Learning Based Remaining Useful Life Prediction Methods for Equipment}

\section{PEI Hong HU Changhua SI Xiaosheng ZHANG Jianxun PANG Zhenan ZHANG Peng}

(College of Missile Engineering, Rocket Force University of Engineering, Xi’an 710025)

\begin{abstract}
With the development of science and technology as well as the advancement of production technology, contemporary equipment is increasingly developing towards large-scale, complex, automated and intelligent direction. In order to ensure the safety and reliability of equipment, the remaining useful life (RUL) prediction technology has received widespread attention and been widely used. Traditional statistical data-driven methods are obviously influenced by the choice of models. Machine learning has powerful data processing ability, and does not need exact physical models and prior knowledge of experts. Therefore, machine learning has a broad application prospect in the field of RUL prediction. In view of this, the RUL prediction methods based on machine learning are analyzed and expounded in detail. According to the depth of machine learning model structure, it is divided into shallow machine learning methods and deep learning methods. At the same time, the development branches and research status of each method are sorted out, and the corresponding advantages and disadvantages are summarized. Finally, the future research directions of RUL prediction methods based on machine learning are discussed.
\end{abstract}

Key words: remaining useful life prediction; machine learning; neural network; support vector machine; deep learning

\section{0 前言}

随着科学技术的发展和生产工艺的进步, 当代 设备的自动化和复杂化程度得到了日益提升。这类 设备在受到内部因素和外部因素的综合作用下，性 能与健康状态难免会呈现出衰退的趋势, 当衰退达 到一定程度时, 设备将无法完成正常任务和功能, 进而造成难以挽回的经济损失和资源浪费 ${ }^{[1-2]}$ 。因而

* 国家自然科学基金(61833016, 61573365, 61773386, 61603398, 61374126, 61473094)和中国科协青年人才托举工程(2016QNRC001)资助项目。 20180912 收到初稿, 20190225 收到修改稿
迫切需要在线掌握设备的健康状态以及切实增强设 备运行的安全性。预测与健康管理(Prognostics and health management, PHM)作为保障设备安全性和可 靠性的一项关键技术，在过去几十年间取得了丰硕 的理论成果并且得到了广泛的实际应用 ${ }^{[3-5]}$ 。在军事 领域中, 作为 PHM 的倠形, 20 世纪美军装备的直 升机健康与使用监测系统(Health and usage monitoring system, HUMS) 已初步具备 PHM 的相关功能。PHM 的理念是针对 20 世纪 70 年代航空航天设备经常面 临高故障率的问题而提出的, 并且经过了多国军方 的共同努力, 这一理念成功应用于联合攻击机 F-35 的项目中 ${ }^{[6]}$ 。现阶段, 美国国防部采购军事装备时 
通常会重点关注装备是否自带 PHM 系统以及 PHM 能力的大小, 可见 PHM 技术在军事领域长期处于 极其重要的地位。在工业领域中, PHM 技术不仅能 够有效降低维修成本、减少停机时间, 而且能够保 障设备的可靠性、提高任务的完成率 ${ }^{[7-8]}$ 。在学术领 域中, 马里兰大学 ${ }^{[3,7]}$ 、佐治亚理工学院 ${ }^{[9]}$ 、清华大 学 ${ }^{[10]}$ 以及国防科技大学 ${ }^{[11]}$ 等相关学术机构均开展 了 PHM 技术的理论研究, 同时结合实际工程问题 不断丰富 PHM 技术理论体系。

PHM 技术主要包含两个部分：剩余寿命 (Remaining useful life, RUL) 预测和健康管理。RUL 通常用于描述当前时刻与失效时刻之间的时间间 隔, 可定义为 ${ }^{[12]}$

$$
T-t \mid T>t
$$

式中， $T$ 表示设备的失效时刻, $t$ 表示当期时刻。 RUL 预测对维修决策和备件订购具有指导性价值, 长期以来被当作 PHM 技术的基础和核心。实现 RUL 预测的主要思想在于根据设备的失效机理、状态监 测数据以及失效数据等有效信息, 确定出 RUL 的分 布或者期望 ${ }^{[13]}$ 。经过了几十年的发展, RUL 预测方 法的研究表现出多样性、体系性、普适性的特点, 研究成果十分丰硕, 同时大批学者致力于对 RUL 预测方法进行分类和总结的研究。HENG 等 ${ }^{[14]}$ 将旋 转机械设备的剩余寿命预测方法分为三类: 传统的 可靠性方法、基于监测数据的方法以及二者融合的 方法, 重点讨论了基于监测数据的方法, 但实际工 程中仍存在大量设备并非属于旋转机械的类别, 如 电子设备 ${ }^{[15]}$ 、高温锅炉 ${ }^{[16]}$ 等。PECHT 等 ${ }^{[7]}$ 以电子设 备为背景, 将剩余寿命预测方法分为: 基于失效机 理分析方法、数据驱动的方法和融合的方法。基于 失效机理分析方法主要通过构建数学模型描述设备 的失效机理, 结合特定设备的经验知识和缺陷增长 方程实现设备的剩余寿命预测 ${ }^{[17-18]}$ 。基于失效机理 分析方法能够准确预测设备的剩余寿命。但需要根 据具体设备进行具体分析, 难以得到推广, 同时随 着设备的复杂性不断提高, 一般很难得到设备的失 效机理或者获得失效机理的代价过高。根据文献 [7], 数据驱动的方法可分为基于机器学习(Machine learning, ML)的方法和统计数据驱动方法, 是当前 剩余寿命预测的主流方法。融合的方法是指失效机 理分析与数据驱动模型相结合方法, 虽然能够充分 利用两种方法的优势, 但过程较为复杂, 因而此类 方法鲜有报道 ${ }^{[19]}$ 。SI 等 ${ }^{[20]}$ 对统计数据驱动的方法进 行了系统的综述, 将其分为两类: 基于直接监测数 据的方法和基于间接监测数据的方法。LIAO 等 ${ }^{[21]}$
将预测模型分为基于经验模型、数据驱动模型和基 于物理模型, 根据三种模型的不同组合重点研究了 混合预测方法的分类和发展历程。除此之外, 专家 学者们根据退化模型不同, 也分别针对特定剩余寿 命预测方法进行了详细而完整的分析, 如基于

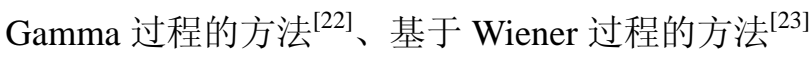
等。上述文献均从不同的角度研究了剩余寿命预测 方法的适用范围和发展现状, 但大部分方法均假定 退化模型是事先知道的, 直接利用状态监测数据或 环境数据对模型参数进行离线或在线估计。然而实 际工程中的退化模型是未知的, 且不同类型退化设 备的退化模型也不尽相同, 退化模型的选择不当将 严重影响剩余寿命的预测精度。基于 ML 的方法能 够克服退化模型未知的问题, 同时构建模型的输入 也不仅局限于状态监测数据, 可以是多种不同类型 的数据。

作为人工智能的核心, ML 的主要功能是使得 计算机模拟或实现人类的学习行为, 通过获取新 的信息, 不断对模型进行训练以提高模型的泛化 能力 ${ }^{[24]}$ 。由于 ML 具有强大的数据处理能力, 该方 法广泛应用于数据挖掘、语音识别、计算机视觉、 故障诊断与寿命预测等领域。随着工业 4.0 时代的 到来, 作为 ML 的一类典型方法, 深度学习正如火如 茶地发展, 为 ML 领域又开辟了一个崭新的方向 ${ }^{[25]}$ 。 基于 ML 的剩余寿命预测方法是剩余寿命预测方法 的一个重要分支, 能够较为准确地对剩余寿命进行 预测。然而, 尽作者所知, 现阶段有关基于 ML 的 设备剩余寿命预测方法综述仍较为贵乏。因而, 本 文对基于 ML 的设备剩余寿命预测方法进行了系统 和完整的综述, 意味着弥补了这项空白。

鉴于此, 根据 ML 模型结构的深度, 将基于 ML 的剩余寿命预测方法分为两类: 基于浅层 ML 的方法和基于深度学习的方法。基于浅层 ML 学习 的方法主要涵盖基于神经网络的方法和基于支持向 量机(Support vector machine, SVM)的方法。基于神 经网络的方法主要包括基于多层感知器(Multi-layer perceptrons, MLP)神经网络的方法、基于径向基函 数(Radial basis function, RBF)神经网络的方法以及基 于极限学习机(Extreme learning machines, ELMs)的 方法。基于深度学习的设备剩余寿命预测方法研究 主要包括: 基于深度神经网络(Deep neural network, DNN)的方法、基于深度置信网络(Deep belief network, $\mathrm{DBN}$ ) 的方法、基于卷积神经网络 (Convolutional neural network, CNN)的方法以及基于递归神经网络 (Recurrent neural network, RNN)的方法。基于 ML 的 剩余寿命预测方法分类框架如图 1 所示。 


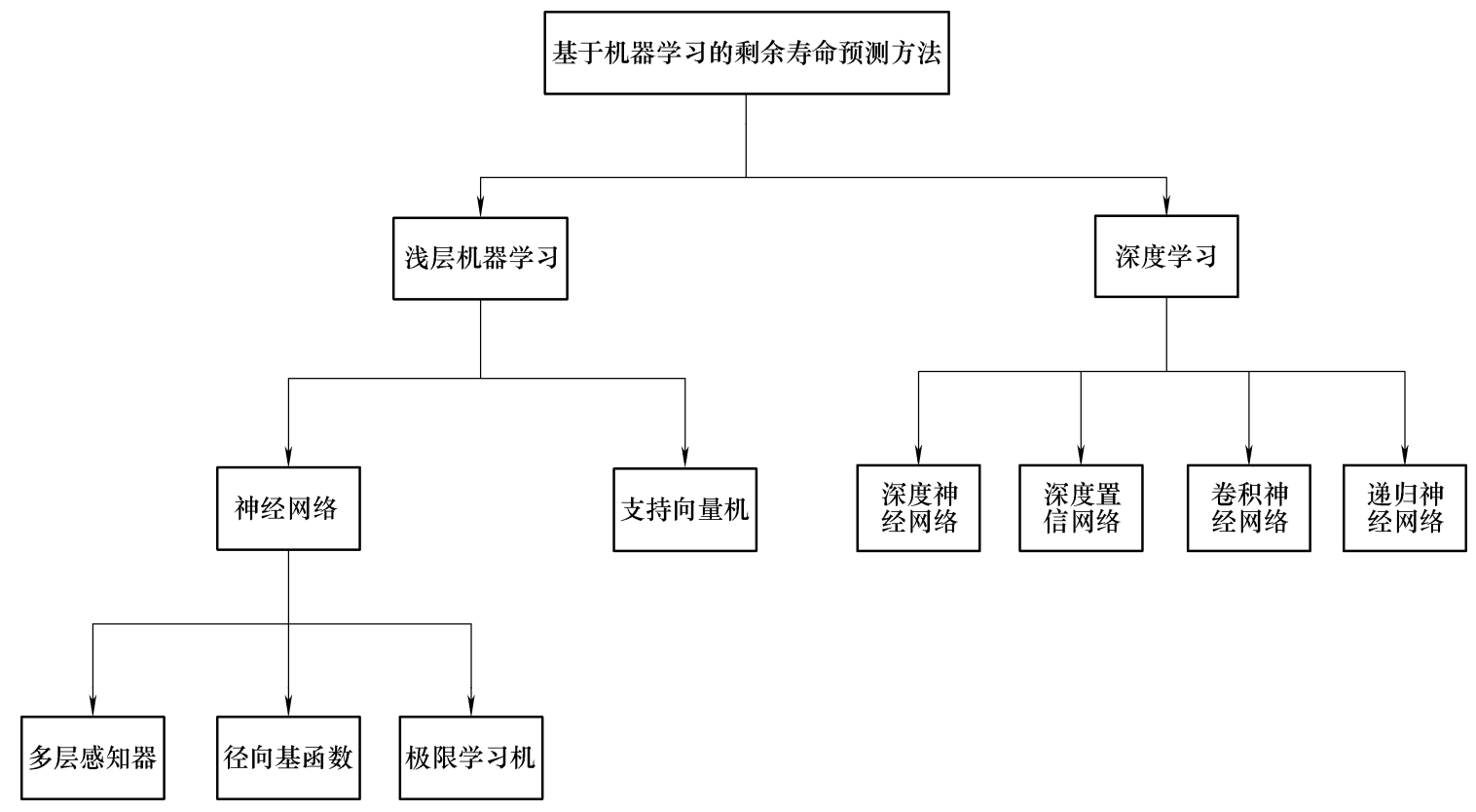

图 1 基于 ML 的设备剩余寿命预测方法分类框架

\section{1 基于浅层 ML 的剩余寿命预测方法}

\section{1 基于神经网络的剩余寿命预测方法}

神经网络作为一种模拟生物神经系统的结构和 功能的数学处理方式, 具有自动学习和总结的能力。 其主要包含输入层、隐含层及输出层, 常用于解决 分类、回归等问题 ${ }^{[28]}$ 。经过了多年来的研究和探索, 在剩余寿命预测领域展现出了强大的优势。基于神 经网络的剩余寿命预测方法旨在以原始测量数据或 基于原始测量数据所提取的特征作为神经网络的输 入, 通过一定的训练算法不断调整网络的结构和参 数, 利用优化后的网络在线预测设备的剩余寿命, 预测过程中无需任何先验信息，完全基于监测数据 得到的预测结果 ${ }^{[29]}$ 。当前基于神经网络的方法主要 包含基于 MLP 神经网络的方法、基于 RBF 神经网 络的方法以及基于 ELMs 的方法。

\subsection{1 基于 MLP 神经网络的剩余寿命预测方法}

MLP 是一类存在隐含层的前馈神经网络, 且隐 含层与输出层的神经元模型是一致的。由于 MLP 通过增加隐藏层或隐藏单元, 具有逼近任意形式非 线性函数的能力, 在剩余寿命预测领域受到了学者 们的广泛关注。MLP 大多采用向后传播 (Back-propagation, BP)算法进行训练。需要说明的 是, 学术界采用 BP 算法训练 MLP 神经网络的方法 通常称为基于 BP 神经网络的方法。尽作者所知, 文献[30]较早开展了基于 BP 神经网络的剩余寿命 预测方法的研究, 并且与自回归移动平均 (Auto-regressive moving average, ARMA)方法进行
了对比分析, 试验结果验证了基于 BP 神经网络的 剩余寿命预测方法具有更优的长期预测能力。此后, GEBRAEEL 等 ${ }^{[31}$ 基于 $\mathrm{BP}$ 神经网络建立了单个轴承 和批量轴承的寿命预测模型，充分利用了寿命周期 内的振动监测信息, 以一定权重融合各轴承指数回 归模型参数, 同时提出了一种参数在线更新方法, 有效保证了寿命预测的准确性。张慰等 ${ }^{[32]}$ 基于 $\mathrm{BP}$ 人工神经网络, 建立了多应力恒定加速寿命试验寿 命预测模型，以加速寿命试验中的加速应力水平和 通过经验分布得到的可靠度作为网络训练输入向 量, 建立了 3 层 BP 神经网络, 进而预测出正常应 力水平下的失效时间。由于在训练过程中, 单一 $\mathrm{BP}$ 神经网络会面临权重陷入局部最优, 以及收玫速度 过慢的问题, 因而学者们通常采用 BP 神经网络与 其他方法相结合的方法对 BP 神经网络进行改进, 以提高剩余寿命的预测准确性 ${ }^{[33]}$ 。WANG 等 ${ }^{[33]}$ 采用 时间序列分析和 BP 神经网络相结合的方法对冷却 风机进行剩余寿命预测, 时间序列分析能够预测数 据变化的趋势, BP 神经网络实时调整预测误差, 保 证了剩余寿命预测精度。文献[34]研究了一种遗传 算法-BP 神经网络的刀具剩余寿命方法, 采用遗传 算法对 BP 神经网络的权重进行全局优化，结果表 明遗传算法-BP 神经网络方法优于单一的 BP 神经 网络方法。SANTHOSH 等 ${ }^{[35]}$ 采用人工神经网络和 威布尔理论对电缆的可靠性和寿命分布进行了预 测, 通过不同温度下的加速寿命数据对人工神经 网络进行训练, 试验分析表明人工神经网络预测 得到的寿命数据与实际寿命数据之间具有较好的 一致性。 
除了采用 BP 算法训练 MLP 外, 同时也会采用 其他方法进行训练, 如文献[36-37]。BEZAZI 等 ${ }^{[37]}$ 采用 MLP 人工神经网络对复合材料结构监测数据 进行建模, 通过极大似然估计和 Bayesian 推理对网 络进行训练, 结果表明该网络具有较好的泛化能力。 在此基础上, PIERCE 等 ${ }^{[38]}$ 基于间隔不确定性技术 进一步分析了该网络的鲁棒性。此类研究为基于 MLP 神经网络训练提供了另外一种思路, 相比于 BP 神经网络而言, 在剩余寿命预测领域研究成果仍 相对贵乏, 有待进一步深入研究。

\subsection{2 基于 RBF 神经网络的剩余寿命预测方法}

RBF 神经网络是 20 世纪 80 年代提出的一种神 经网络结构, 具有单隐层的三层前馈网络, 且能够 以任意精度逼近任意连续非线性函数 ${ }^{[39-40]}$ 。 $\mathrm{RBF}$ 神 经网络在结构上与 MLP 神经网络最大的区别在于 激励函数的自变量为输入矢量与权值矢量之间距离 与偏差的乘积, 而非输入矢量与权值矢量之间的加 权和。由于 RBF 神经网络具有输出与初始权值无 关、训练时间较短等的优良特性, 近年来 RBF 神经 网络已成功应用于信号处理、系统建模、过程控制、 故障诊断与寿命预测等领域, 并表现出了非凡的优 势。文献[41]指出, RBF 神经网络模型的关键在于 正确选择合适的 RBF 中心, 隐含层 RBF 中心的数 目和位置直接影响了网络的近似能力。由于文献[32] 采用的 BP 神经网络会面临计算复杂、训练速度过 慢的问题, LI 等 ${ }^{[42]}$ 对文献[32]中的方法进行了改进, 采用灰色 $\mathrm{RBF}$ 神经网络的方法构建了加速寿命试 验寿命预测模型, 试验表明预测结果明显优于 BP 神经网络。李志刚等 ${ }^{[43]}$ 采用 $\mathrm{RBF}$ 神经网络用于继电 器寿命预测时, $\mathrm{RBF}$ 神经网络的输入信息并非是原 始的具有非平稳特征的继电器超程时间, 而是通过 小波变换后得到的随机项, RBF 神经网络的输出信 息再次通过小波包重构实现了继电器的寿命预测。 CHEN 等 ${ }^{[44]}$ 提出一种多元灰色 RBF 混合模型用于 工业设备的剩余寿命预测, 融合了灰色模型和 RBF 神经网络的优点, 有效保证了预测精度, 具有实际 工程应用价值。

与基于 BP 神经网络的剩余寿命预测方法相比, 一方面, 基于 RBF 神经网络的剩余寿命预测方法的 网络仅包含一个隐含层, 而 BP 神经网络包含多个 隐含层, 隐含层的数目直接决定了拟合精度, 因而 $\mathrm{BP}$ 神经网络层数的确定需要增加额外的时间或需 要根据工程实践经验; 另一方面, $\mathrm{BP}$ 神经网络容易 陷入局部最优且学习过程收玫过慢, 基于 RBF 神经 网络的剩余寿命预测方法能够克服这类问题, 可实 现网络结构和隐层单元的数据中心的动态确定, 具
有自适应预测剩余寿命的能力。

\subsection{3 基于 ELMs 的剩余寿命预测方法}

ELMs 作为一种针对单隐含层前馈神经网络的 新型学习算法, 是新加坡学者黄广斌于 2004 年提出 的 ${ }^{[45]}$ 。ELMs 训练过程的基本思路在于随机选择输 入权值和隐含层偏差值, 根据工程实践经验人工选 择隐含层神经元个数, 通过最小二乘方法确定输出 权值, 实现了网络结构和参数的快速确定。由于 ELMs 有着学习速度快、泛化能力强等特点, 目前 在工程设备的故障诊断、寿命预测与可靠性评估等 领域中引起了学者和工程人员的普遍关注。李䂞 ${ }^{[46]}$ 基于 ELMs 研究了风机机械传动部件的剩余寿命预 测问题, 详细介绍了 ELMs 算法的原理、参数选取 和优化过程, 进而能够预测出相关性能参数的趋势 和数值, 实现性能参数的评估和剩余寿命的预测。 LIU 等 ${ }^{[47]}$ 通过两层特征矩阵的联合近似对角化方法 提取出能够更好反映轴承退化过程的特征, 将提取 后的特征输入 ELMs 模型, 实现了轴承剩余寿命的 准确预测。在此基础上, 文献[48]对该特征提取方 法进行了改进，同样采用 ELMs 方法对提取后的特 征进行训练, 并且将该方法应用于轴承的剩余寿命 研究中。杜占龙等 ${ }^{[49]}$ 基于 sigmoid 后验概率映射和 Lagrange 成对耦合法提出了一种多分类概率 ELMs 模型, 解决了无人机发射机寿命剩余预测问题。 YANG 等 ${ }^{[50]}$ 提出了一种基于 ELMs 的剩余寿命预测 方法, 对比了 ELMs 与 BP 人工神经网络两者间的 联系与区别, 发现 ELMs 的内部参数无需迭代计算, 试验表明基于 ELMs 的模型在预测的精确度和稳定 性方面稍劣于基于 BP 人工神经网络的模型, 但却 能显著降低训练时间。

由于采用 ELMs 方法对输入权值、隐含层偏差 值以及隐含层神经元个数选择时会存在一定的随机 性, 难以保证预测精度, 因而学者们开始致力于对 基于 ELMs 的剩余寿命预测方法进行改进和优化。 根据 ELMs 基本结构, ELMs 仅有一个隐含层, 隐 含层内的神经元直接决定了预测模型的性能, 为避 免隐含层神经元的数目选择问题, WANG 等 ${ }^{[51]}$ 提出 了一种带有核的 ELMs, 实现了非平稳序列的在线 预测。针对存在多个性能指标的工程轴承而言, 何 群 等 ${ }^{[52]}$ 通过主成分分析 (Principal component analysis, PCA)技术消除多个特征间的午余性和相关 性, 同时在单变量 ELMs 的基础上构建了多变量 ELMs 预测模型, 克服了传统单变量极限学习机结 构简单、信息贵乏的缺点, 有效提高了轴承剩余寿 命的预测精度。针对 ELMs 网络中的输入权值和隐 含层偏差值为随机产生, 无法保证 ELMs 的网络训 
练效果的问题, 吴婧睿等 ${ }^{[33]}$ 采用萤火虫算法确定出 最优的输入权值和隐含层偏差值, 保证 ELMs 模型 具有最小化的输出权重范数, 同时将此方法应用于 Li 电池的剩余寿命预测, 提高了预测模型的精确性 和稳定性。

基于 ELMs 的剩余寿命预测方法优势在于:

(1) 能够实现快速剩余寿命预测, 有效减少了模型训 练时间; (2) 激活函数可使用不连续的函数; (3) 避 免了采用梯度下降学习算法中学习参数选择敏感和 易陷入局部极值的问题。尽管基于 ELMs 的方法具 有诸多优势, 但同时也存在一定的不足。由于输入 权值与隐含层偏差值为随机产生的, 无法保证 ELMs 的网络训练效果, 可能会时好时坏; 同时隐 层节点数需根据经验及试验的方法进行选择, 难以 保证得到最优模型。另外, 由于输出权值通过最小 二乘法计算得到, 因而基于 ELMs 的方法会面临扩 大离群点与噪声影响的难题。

\section{2 基于 SVM 的剩余寿命预测方法}

作为一种新的 ML 方法, SVM 是基于 VC 维理 论和结构风险最小化原理发展起来的。SVM 是由 Cortes 和 Vapnik 于 1995 年首次提出的, 主要用于 解决 ML 的分类和回归问题, 适用于分析小样本和 多维化数据 ${ }^{[54-55]}$ 。而后, SVM 开始受到可靠性工程 领域专家学者的普遍关注 ${ }^{[56-57]}$ 。基于 SVM 的剩余 寿命预测方法研究主要思路是利用实际工程中获得 的状态监测数据训练支持向量机模型, 确定出模型 参数(不敏感系数、惩罚因子、核函数参数等), 基 于训练后的 SVM 模型对系统未来的状态进行预 测, 通过与预先设定的失效阈值对比得到设备的剩 余寿命。

由于实际工程中状态监测数据具有多维性、非 线性以及不确定性等特征, 单纯采用 SVM 方法训 练状态监测数据通常难以保证 SVM 模型参数的准 确性, SVM 模型参数直接影响了设备剩余寿命结 果, 因而学者们开始关注如何将 SVM 通常与其他 方法结合起来对设备剩余寿命进行预测。为剔除数 据中存在的干扰信息, MIAO 等 ${ }^{[58]}$ 将小波分析与 SVM 结合起来用于陀螺仪的剩余寿命预测。NIETO 等 ${ }^{[59]}$ 提出了一种基于混合粒子群优化和 SVM 的算 法用于航天器发动机剩余寿命预测, 解决了支持向 量机训练过程中超参数的优化问题, 进一步提高了 预测的准确性。针对 SVM 难以处理具有单调趋势 的非静态序列问题, MAIOR 等 ${ }^{[60]}$ 提出了一种经验 模态分解与 SVM 相结合的方法用于退化数据分析 和剩余寿命预测, 并且将所提方法应用于电动机的 实例分析中, 结果表明与单纯 SVM 相比, 该方法
能够改善预测性能。CHEN 等 ${ }^{[61]}$ 采用 SVM 对参考 设备的剩余寿命进行预测, 并且基于改进后的相似 性理论结合参考设备的剩余寿命结果对运行设备的 剩余寿命进行预测, 为考虑参考设备在各采样点的 信息对运行设备剩余寿命的不同影响, 在分析过程 中引入权重可调系数, 最后将所提方法应用于航空 发动机的剩余寿命评估。

通过上述分析可以看到, 尽管采用 SVM 能够 有效解决一些工程设备的剩余寿命问题, 但同样会 存在诸多缺陷。如: (1) 随着样本集的增大, 线性将 增加, 进而造成过拟合和计算时间的增加; (2) 难以 得到概率式的预测, 即无法评估剩余寿命预测的不 确定性; (3) 核函数必须满足 Mercer 条件。为有效 解决或减少 SVM 的固有缺陷, TIPPING 等 ${ }^{[62]}$ 在 Bayesian 框架下提出了相关向量机(Relevance vector machine, RVM) 方法。学者们陆续开展有关 RVM 的 理论研究, 并与剩余寿命预测领域相关联。MAIO 等 ${ }^{[63]}$ 提出了一种融合 RVM 和指数回归的剩余寿命 预测方法, 适用于分析信噪比较低且包含竞争退化 过程的数据, 克服了过拟合的难题, 同时将所提方 法用于预测推力轴承的剩余寿命。SONG 等 ${ }^{[64]}$ 采用 RVM 对电池容量进行训练和预测, 同时利用卡尔曼 滤波对相关向量机的输出进行在线优化, 进而实现 了电池的剩余寿命预测, 该方法考虑了系统噪声和 测量不确定性因素的影响, 克服了 RVM 无法长期 预测的短板。为应对 SVM 训练速度慢、存储量大 的难题, 学者们引入了最小二乘支持向量机(Least square support vector machine, LS-SVM), 将求解二 次规划问题转化为求解高维空间线性最小二乘问 题, 简化了计算过程, 提高了训练速度。王烨等 ${ }^{[65]}$ 将 Bayesian 推断应用于 LS-SVM 模型参数选择中, 优化了 LS-SVM 模型参数, 构建了发动机在翼寿命 的非线性预测模型，同时与 BP 神经网络、遗传算 法、普通网格搜索进行了对比, 结果表明所提方法 具有更好的泛化能力和预测精度。

\section{2 基于深度学习的剩余寿命预测方法}

随着 “大数据、云计算” 时代的到来以及传 感器技术的发展, 工业系统中能够获取的监测数 据越来越多。传统的浅层 ML 算法很大程度上依 赖于专家先验知识与信号处理技术, 难以自动处 理和分析海量的监测数据。而深度学习作为神经 网络发展而来的一项新技术, 以其强大的特征提 取能力为训练海量数据提供了一种解决思路。2006 年, 由 HINTON 等 ${ }^{[66]}$ 提出的贪婪逐层预训练方法 
实现了深度学习在理论上的突破, 随后大批学者开 始了对深度学习方法的探索与研究。经过了十余年 的探索, 深度学习当前已成功应用在许多工程领域, 如图像识别 ${ }^{[67]}$ 、语音识别 ${ }^{[68]}$ 、故障诊断 ${ }^{[69]}$ 、寿命预 测 ${ }^{[70]}$ 等。ZHAO 等 ${ }^{[71]}$ 分别讨论了几种深度学习方法 的原理, 如自编码器(Auto-encoders, AE)及其变形、 $\mathrm{DBN} 、 \mathrm{CNN}$ 等, 同时分析了其在机械健康监测领 域的广泛应用, 但并未涉及在剩余寿命预测领域 的研究现状。本文在文献[71]基础上, 重点阐述基 于深度学习的剩余寿命预测方法研究成果。根据 深度学习网络结构的不同, 基于深度学习的剩余 寿命预测方法研究主要包括：基于 DNN 的方法、 基于 $\mathrm{DBN}$ 的方法、基于 $\mathrm{CNN}$ 的方法以及基于 $\mathrm{RNN}$ 的方法。

\section{1 基于 DNN 的剩余寿命预测方法}

DNN 通常是由多层特征表示模型堆叠形成的 多层神经网络, 常见的特征表示模型有 $\mathrm{AE}$ 以及降 噪自动编码器(Denoising autoencoder, $\mathrm{DAE}$ )等。 $\mathrm{AE}$ 主要包含编码器和解码器两部分, 编码器将输入数 据从高维空间映射到低维空间, 具有获取输入数据 特征表示的能力, 解码器能够将输入数据从低维空 间映射到高维空间, 能够对输入进行复现 ${ }^{[72]}$ 。当监 测数据受到噪声干扰时, AE 所提取特征的鲁棒性 较差, 因而通过给予 $\mathrm{AE}$ 一定的约束而提出了 $\mathrm{DAE}$, 降低了对随机扰动的敏感性。基于 DNN 的方法主 要思路在于通过多个 $\mathrm{AE}$ 或 $\mathrm{DAE}$ 堆叠网络提取出原 始数据的高层次特征，进而基于回归拟合方法或前 馈神经网络实现剩余寿命的预测。雷亚国等 ${ }^{[73]}$ 将多 个 DAE 层层堆叠构成深度神经网络的隐层结构, 以 机械设备的频域信号作为输入对 DNN 进行预训练 和微调, 实现了故障特征的自适应的提取和健康状 态的智能诊断, 克服了传统特征提取与故障识别方 法依赖于信号处理技术与专家经验知识, 但未涉及 剩余寿命预测的研究。周福娜等 ${ }^{[74]}$ 提出了一种基于 DNN 的微小缓变故障早期诊断方法, 通过 PCA 方 法将深度学习所提取的高维故障特征转换成一维故 障特征，进而利用非线性拟合方法构建出寿命预测 模型。在文献[74]的基础上, 王佳瑜 ${ }^{[75]}$ 将非线性拟 合方法替换为 BP 神经网络进行训练。YAN 等 ${ }^{[76]}$ 针 对工业系统大数据分析情形, 研究了一种深度 DAE 与回归分析相结合的剩余寿命预测方法, 用两个深 度 DAE 分别对远端信号与近端信号进行处理得到 整体趋势和当前变化过程, 融合两个深度 DAE 的输 出, 通过线性回归预测出设备的剩余寿命。

基于 DNN 的剩余寿命预测方法具有以下特点: (1) 通过输入数据的多次降维可实现有用特征的准
确提取, 能够方便模型的训练; (2) 由于 DAE 具有 降噪滤波功能, 多个 DAE 堆叠形成的网络能够处理 包含噪声的监测数据, 充分体现出该方法具有强大 的鲁棒性和普适性。

\section{2 基于 DBN 的剩余寿命预测方法}

DBN 作为一种典型的深度学习方法, 主要由多 个受限波尔兹曼机(Restricted Boltzman machine, RBM) 堆叠与一个分类层或回归层组合形成的深度网络, 不仅能够实现观测数据从低级到高级的特征表示与 提取, 而且能够发现输入数据的分布式特征 ${ }^{[77]}$ 。 DBN 一般通过逐层贪婪的前向学习与梯度下降的 反向微调相结合的算法对模型进行训练。堆叠 RBM 主要完成对输入数据的特征提取, 通常选择对比散 度算法预先确定网络的参数, 分类层/回归层是通过 非线性函数将前面提取到的高层次特征映射到标签 上，便于 $\mathrm{DBN}$ 的反向调整。由于 $\mathrm{DBN}$ 具有强大的 特征提取能力, 有效解决了人工特征提取与选择所 引起不确定的问题, 实现了智能特征提取的目标, 同时该方法下的时域信号无需满足周期性的要求, 因而在剩余寿命预测领域具有广阔的应用空间。

单基于深度置信网络对发动机的健康状态进 行在线监测, 给出了权值和偏置的更新公式, 实例 表明在故障诊断性能方面 DBN 明显优于 BP 神经网 络 ${ }^{[78]}$ 。DEUTSCH 等 ${ }^{[79]}$ 将 DBN 成功应用于预测轴 承的剩余寿命中，但所提方法的预测精度远低于粒 子滤波方法。DEUTSCH 等 ${ }^{[80]}$ 提出了一种融合 DBN 与前馈神经网络(Feedforward neural network, FNN) 的旋转设备剩余寿命预测方法, 这是基于 DBN 方 法的改进和拓展, 能够有效结合 DBN 特征提取能 力与 FNN 的预测性能。基于此, 为得到剩余寿命的 概率分布, 将 DBN 与粒子滤波有效结合起来, 进 一步提高了预测精度 ${ }^{[81]}$ 。张国辉 ${ }^{[22]}$ 深入研究了 $\mathrm{DBN}$ 的基本原理、预测模型以及网格参数影响, 将其成 功应用于轴承的剩余寿命预测, 同时针对深度置信 网络预测结果无法表达不确定度的问题, 率先提出 了一种融合 DBN 与 SVM 的 Li 电池剩余寿命预测 方法, 不仅有效提高了预测精度, 而且给出了置信 区间和概率密度函数。在此基础上, ZHAO 等 ${ }^{[83]}$ 有 效结合了 $\mathrm{DBN}$ 与 RVM 二者的优势, 研究了一种新 的 Li 电池剩余寿命预测方法。ZHANG 等 ${ }^{[84]}$ 对传统 深度置信网络进行了改进, 提出了一种多目标深度 置信网络集合方法, 每个深度置信网络的输出对网 络集合的输出均占有一定权重, 同时试验表明所提 方法在分析 NASA 的航空发动机数据时表现出了 强大的优越性。

尽管 DBN 在剩余寿命预测领域表现出强大的 
优势, 但也存在一定的不足: (1) DBN 的短期预测 性能较好, 而长期预测性能较差; (2) 基于 DBN 得 到的剩余寿命预测结果无法反映出预测结果的不确 定性, 一般需要与其他方法相结合进行预测, 以体 现出预测结果的不确定度。因而现有基于 DBN 的 剩余寿命预测方法研究侧重于在克服 DBN 劣势的 基础上, 对 DBN 进行改进以达到预期的剩余寿命 预测精度。

\section{3 基于 CNN 的剩余寿命预测方法}

CNN 作为一类经典的前馈神经网络, 是由 LeCun 首次提出并用于解决图像处理问题的, 主要 由若干卷积层和池化层组成。其目的在于通过构建 多个滤波器, 逐级提取出隐藏在监测数据中的拓扑 结构特征, 提取的特征会随着网络层次的深入而愈 加抽象 ${ }^{[85]}$ 。对于 CNN 而言, 卷积层利用原始输入 数据对多个局部滤波器进行卷积处理, 随后的池化 层能够以固定长度提取最为重要的特征, 经常采用 的池化函数为最大池化函数 ${ }^{[86]}$ 。由于 CNN 模型具 有特殊的网络结构, 其不仅能够输入序列数据, 而 且能处理网格化数据, 在计算机视觉、语音识别等 领域应用十分广泛。

虽然 CNN 具有稀疏交互、参数共享、空间池 化等优良特性, 同时在很多领域具有广阔的应用空 间, 但基于 CNN 实现剩余寿命预测的研究是从 2016 年才开始的。BABU 等 ${ }^{[87]}$ 将深度 CNN 应用于剩余 寿命预测领域, 采用两个卷积层和两个池化层提取 原始信号的特征, 同时结合 MLP 实现剩余寿命的预 测。LI 等 ${ }^{[8]}$ 提出了一种基于深度 $\mathrm{CNN}$ 的多变量设 备剩余寿命估计方法, 为更好地提取特征, 这里采 用时间窗方法用于获得样本, 同时由于部分有效信 息将被池化操作所滤除, 在构建网络过程中忽略了 池化层。 REN 等 ${ }^{[89]}$ 基于 CNN 研究了轴承剩余寿命 预测的问题, 将所提取的一系列特征, 即频谱主能 量向量组合成特征图, 通过 CNN 的结构能够提取 出有助于预测剩余寿命的一维矢量, 将此一维矢量 输入深度神经网络实现剩余寿命的预测, 轴承试验 表明所提方法优于传统的 ML 方法。

基于 $\mathrm{CNN}$ 的剩余寿命预测研究具有以下特点: (1) $\mathrm{CNN}$ 特别适用于能够监测到海量数据的工程设 备; (2) 能够实现自动特征提取与识别, 无需人工参 与和干预; (3) 权重共享特性使得 CNN 模型的参数 数量较少, 优化过程较为方便。但基于 CNN 的剩 余寿命预测研究起步较晚, 当前仍处于初步探索阶 段, 研究成果尚未成体系, 同时无法定量给出剩余 寿命的不确定度, 因而, 基于 CNN 的方法仍需深 入的研究。

\section{4 基于 RNN 的剩余寿命预测方法}

RNN 为一类包含前馈连接与内部反馈连接的 前馈神经网络, 主要用于处理具有相互依赖特性的 监测向量序列。由于其特殊的网络结构, 能够保留 隐含层上一时刻的状态信息, 因而在对复杂动态系 统建模领域表现出了强大的优势 ${ }^{[90]}$ 。基于 RNN 的 剩余寿命预测方法基本思路为将工程中的监测数据 输入作为 RNN 网络的输入, 采用通过时间反向传 播(Back-propagation through time, BPTT)对模型参 数进行训练, 进而实现设备的剩余寿命预测。需要 说明的是, RNN 的内部反馈连接刻画了监测数据的 前后依赖性。

经过专家学者们的不解努力, 基于 RNN 的剩 余寿命预测方法研究成果丰硕, 应用也十分广泛。 HEIMES $^{[91]}$ 基于 RNN 结构实现了剩余寿命的预测, 通过 BPTT 算法与扩展卡尔曼滤波方法对模型进行 训练, 试验表明所提方法性能明显优于基于 MLP 的方法。针对环境与负载具有时变性问题, LIU 等 ${ }^{[22]}$ 采用自适应 RNN 对 Li 电池的剩余寿命进行预测, 通过循环 Levenberg-Marquardt方法在线优化网络结 构的权重。MALHI 等 ${ }^{[93]}$ 选择竞争学习方法对输入 数据进行集群处理, 将处理后的数据输入 RNN 网 络, 不仅提升了网络训练速度, 而且改善了长期预 测性能。然而, 传统 RNN 通常存在“记忆衰退”的问 题, 因为在传统循环层中不存在控制记忆流动的结 构, 当处理长时间监测序列时, 将会产生较大的预 测偏差。鉴于此, 学者们对 RNN 在网络模型上进 行了改进, 提出了一种长短期记忆(Long short-term memory, LSTM)模型。LSTM 主要包含两部分内容: (1) 长期状态与短期状态; (2) 三个控制门(遗志门、 输入门、输出门)。门结构作为 LSTM 的独特结构, 能够在最优条件下确定出所通过信息特征 ${ }^{[94]}$ 。WU 等 ${ }^{[94]}$ 将 LSTM 应用于工程设备的剩余寿命预测中, 采用 Dropout 技术提升 LSTM 的泛化能力, 基于 NASA 航空发动机数据库分析了四种不同情形, 预 测效果明显优于传统 RNN 模型。为体现剩余寿命 的概率分布, ZHANG 等 ${ }^{[95]}$ 提出了一种基于 LSTM 的锂电池剩余寿命预测模型, 解决了退化数据长期 依赖型问题, 其长期学习能力优于支持向量机和传 统 RNN, 通过 Monte Carlo 仿真方法得到了剩余寿 命的概率分布。GUO 等 ${ }^{[96]}$ 选择相关相似性(Relatedsimilarity, RS)特征对时频域数据进行预处理, 采用 相关性和单调性度量用于特征选择标准, 基于所选 择的特征对 LSTM 进行训练, 有效克服了梯度消失 或爆炸情形, 同时避免了需要预先人工设定阈值的 问题。为解决运行和环境扰动引起的不确定问题, 
ZHANG 等 ${ }^{[97]}$ 对 LSTM 的结构进行了改进, 研究了 一种基于双向 LSTM 的剩余寿命预测方法, 前向路 径与后向路径独立计算且将输出进行串联, 有效平 滑了退化轨迹和预测结果。

基于 RNN 的剩余寿命预测方法能够融合原有 学习样本与新的学习模式实现样本的重新训练, 不 仅能够提高剩余寿命预测的准确性, 而且具有收玫 速度快和稳定性高等特点, 在可靠性评估和剩余寿 命预测领域发挥着重要作用。值得指出的是, 现有 研究在理论上和应用上仍受到一些限制。一方面, 当处理长期依赖型退化数据时, 传统 RNN 方法会 面临梯度消失或爆炸问题, 剩余寿命预测精度会受 到严重影响。虽然学者们一直致力于对传统 RNN 进行结构上改进以避免此类问题, 仍需要针对此问 题进行进一步研究。另一方面, RNN 在处理时序数 据或前后依赖退化数据具有一定的优势, 但难以对 多维数据进行分析和处理, 通常需要与其他方法结 合进行研究。

\section{3 未来研究方向}

作为 PHM 技术的基础, RUL 预测方法为维修 决策提供可靠的理论依据。随着信息物理系统 (CPS-cyber-physical system, CPS)的广泛应用, 基于 ML 的设备剩余寿命预测方法再次受到了学者们和 工程技术人员们的重点关注。基于 ML 的设备剩余 寿命预测方法不仅能够有效提取出状态监测数据中 能反映出设备健康状态的特征信息, 而且能够刻画 出状态监测数据与输出数据间的非线性关系进而实 现剩余寿命的准确预测。然而根据现有研究成果, 理论上与工程中仍存在大量的挑战与问题有待进一 步研究。下面主要对未来可能的研究方向进行详细 说明。

(1) 多种失效模式下设备剩余寿命预测研究。 现有研究大都侧重于单一失效模式下设备的剩余寿 命预测, 忽略了工程设备的失效是由于多种失效模 式耦合作用所引起的情形, 如机械设备或电子设备 通常会发生退化失效, 但受到外界冲击或电应力影 响下, 设备会突然无法实现正常功能, 这类失效为 突发失效。因而多种失效模式下设备剩余寿命预测 研究值得进一步研究。

(2) 考虑相互影响的多部件设备剩余寿命预测 研究。当前复杂工程设备往往是由多个零部件通过 一定连接方式所组成的, 这些零部件之间的退化过 程和失效模式往往是相互影响的, 共同决定了工程 设备的剩余寿命。如惯性导航系统主要由多个陀螺
仪与加速度计组成, 这些零部件的安装方式、连接 形式、相互作用机制均会影响惯性导航系统的剩余 寿命。但基于 ML 的设备剩余寿命预测方法未能有 效考虑部件间的相互作用, 因而考虑相互影响的多 部件设备剩余寿命预测研究更加符合实际, 需要得 到重点关注。

(3) 智能化特征提取与剩余寿命预测研究。准 确进行剩余寿命预测的前提是从海量的监测数据 中尽可能多的提取出有效信息, 但传统的统计数 据驱动方法与浅层 ML 方法均需要依靠大量信号 处理技术与专家经验知识, 手动提取出特征信息, 对于处理复杂工程设备的海量监测数据而言, 这 些方法受到了严重限制。深度学习能够在一定程 度上克服此类问题, 如 DBN 与 CNN 均具有智能 化特征提取与剩余寿命预测的能力, 但有关智能 化特征提取与剩余寿命预测研究仍较为贵乏, 有 待深入的研究。

(4) ML 与传统统计数据驱动方法相结合的研 究。以神经网络与深度学习为代表的 ML 能够提取 出监测数据中所蕴含的有效信息, 刻画出特征信息 与剩余寿命之间的非线性关系, 在剩余寿命预测领 域具有一定的普适性, 但无法得到剩余寿命的解析 概率分布，难以应用于维修策略的安排和制定; 以 Wiener 过程与 Gamma 过程为代表的传统统计数据 驱动方法可根据退化轨迹估计出退化模型参数, 推 导出剩余寿命的解析概率分布, 但剩余寿命预测精 度受到所选退化模型的影响较大。因而在后续研究 中, 需要考虑如何融合两类方法的优势。

\section{4 结论}

主要对基于 ML 的设备剩余寿命预测方法进行系 统的综述和总结。根据 ML 模型结构的深度, 将其分 为基于浅层 ML 的方法和基于深度学习的方法。同时 疏理了每类方法的发展分支与研究现状, 并且总结了 相应的优势和缺点, 最后探讨了基于 ML 的剩余寿命 预测方法的未来研究方向。

\section{参 考 文 献}

[1] MOGHADDASS R, ZUO M J. An integrated framework for online diagnostic and prognostic health monitoring using a multistate deterioration process[J]. Reliability Engineering and System Safety, 2014, 124: 92-104.

[2] QIAO W, ZHANG P, CHOW M Y. Condition monitoring, diagnosis, prognosis, and health management for wind energy conversion systems[J]. IEEE Transactions 
on Industrial Electronics, 2015, 62(10): 6533-6535.

[3] PECHT M , JAAI R. A prognostics and health management roadmap for information and electronics-rich systems[J]. Microelectronics Reliability, 2010, 50(3): 317-323.

[4] LAU D, FONG B. Special issue on prognostics and health management[J]. Microelectronics Reliability, 2011 , 51(2): 253-254.

[5] ZHAO Z, QUAN Q, CAI KY. A profust reliability based approach to prognostics and health management[J]. IEEE Transactions on Reliability, 2014, 63(1): 26-41.

[6] SMITH G, SCHROEDER J B, NAVARRO S, et al. Development of a prognostics and health management capability for the Joint Strike Fighter[C]//1997 IEEE Autotestcon Proceedings AUTOTESTCON, 2002 : 676-682.

[7] PECHT M. Prognostics and health management of electronics[M]. Hoboken: John Wiley and Sons, Ltd, 2008.

[8] YE Z S, XIE M. Stochastic modelling and analysis of degradation for highly reliable products[J]. Applied Stochastic Models in Business and Industry, 2015, 31(1): 16-32.

[9] GEBRAEEL N, ELWANY A, PAN J. Residual life predictions in the absence of prior degradation knowledge[J]. IEEE Transactions on Reliability, 2009, 58(1): 106-117.

[10] SI X S , WANG $\mathrm{W}, \mathrm{HU} \mathrm{C} \mathrm{H}$, et al. A Wiener-process-based degradation model with a recursive filter algorithm for remaining useful life estimation[J]. Mechanical Systems \& Signal Processing, 2013, 35(1-2): 219-237.

[11] WANG X, BALAKRISHNAN N, GUO B. Residual life estimation based on nonlinear-multivariate Wiener processes[J]. Journal of Statistical Computation and Simulation, 2015, 85(9): 1742-1764.

[12] ZIO E, MAIO F D. A data-driven fuzzy approach for predicting the remaining useful life in dynamic failure scenarios of a nuclear system[J]. Reliability Engineering \& System Safety, 2013，95(1): 49-57.

[13] GEBRAEEL N, PAN J. Prognostic degradation models for computing and updating residual life distributions in a time-varying environment[J]. IEEE Transactions on Reliability, 2008, 57(4): 539-550.

[14] HENG A, ZHANG S, TAN A C C, et al. Rotating machinery prognostics: State of the art, challenges and opportunities[J]. Mechanical Systems \& Signal
Processing, 2009, 23(3): 724-739.

[15] LIU D T, WANG H, PENG Y, et al. Satellite lithium-ion battery remaining cycle life prediction with novel indirect health indicator extraction[J]. Energies, 2013, 6(8): 3654-3668.

[16] ZHANG J X, HU C H, HE X, et al. Lifetime prognostics for deteriorating systems with time-varying random jumps[J]. Reliability Engineering and System Safety, 2017, 167: 338-350.

[17] LI C J, LEE H. Gear fatigue crack prognosis using embedded model, gear dynamic model and fracture mechanics[J]. Mechanical Systems and Signal Processing, 2005, 19(4): 836-846.

[18] FAN J J , YUNG K C , PECHT M. Physics-of-failure-based prognostics and health management for high-power white light-emitting diode lighting[J]. IEEE Transactions on Device and Materials Reliability, 2011, 11(3): 407-416.

[19] CHENG S, PECHT M. A fusion prognostics method for remaining useful life prediction of electronic products[C]//IEEE International Conference on Automation Science and Engineering, 2009: 102-107.

[20] SI X S, WANG W B, HU C H, et al. Remaining useful life estimation : A review on the statistical data driven approaches[J]. European Journal of Operational Research, 2011, 213(1): 1-14.

[21] LIAO L, KÖTTIG F. Review of hybrid prognostics approaches for remaining useful life prediction of engineered systems, and an application to battery life prediction[J]. IEEE Transactions on Reliability, 2014, 63(1): 191-207.

[22] NOORTWIJK J M V. A survey of the application of gamma processes in maintenance[J]. Reliability Engineering \& System Safety, 2009, 94(1): 2-21.

[23] ZHANG Z X, SI X S, HU C H, et al. Degradation data analysis and remaining useful life estimation: A review on Wiener-Process-Based methods[J]. European Journal of Operational Research, 2018.

[24] MITCHELL T M, CARBONELL J G, MICHALSKI R S. machine learning[M]. Beijing: China Machine Press, 2003.

[25] BENGIO S, DENG L, LAROCHELLE H, et al. Guest editors' introduction: Special section on learning deep architectures[J]. IEEE Transactions on Pattern Analysis and Machine Intelligence，2013，35(8): 1795-1797.

[26] YU D, HINTON G, MORGAN N, et al. Introduction to the special section on deep learning for speech and 
language processing[J]. IEEE Transactions on Audio Speech \& Language Processing, 2012, 20(1): 4-6.

[27] LECUN Y, BENGIO Y, HINTON G. Deep learning[J]. Nature, 2015, 521(7553): 436-444.

[28] SIMON H. Neural network : A comprehensive foundation[M]. New Jersey: Prentice Hall PTR, 1994.

[29] BYINGTON C S, WATSON M, EDWARDS D. Data-driven neural network methodology to remaining life predictions for aircraft actuator components[C]//IEEE Aerospace Conference Proceedings, 2004: 3581-3589.

[30] SHAO Y, NEZU K. Prognosis of remaining bearing life using neural networks[J]. Proceedings of the Institution of Mechanical Engineers-Part I, 2000, 214(3): 217-230.

[31] GEBRAEEL N, LAWLEY M, LIU R, et al. Residual life predictions from vibration-based degradation signals: A neural network approach[J]. IEEE Transactions on Industrial Electronics, 2004, 51(3): 694-700.

[32] 张慰, 李晓阳, 姜同敏, 等. 基于 $\mathrm{BP}$ 神经网络的多应 力加速寿命试验预测方法 [J]. 航空学报, 2009, 30(9): 1691-1696.

ZHANG Wei, LI Xiaoyang, JIANG Tongmin, et al. Life-prediction of multi-stress accelerated life testing based on BP algorithm of artificial neural network[J]. Acta Aeronautica et Astronautica Sinica, 2009, 30(9): 1691-1696.

[33] WANG L X, WU Z H, FU Y D, et al. Remaining life predictions of fan based on time series analysis and BP neural networks[C]//nformation Technology , Networking , Electronic and Automation Control Conference, IEEE. 2016: 607-611.

[34] ZHANG Z, LI L, ZHAO W. Tool life prediction model based on GA-BP neural network[J]. Materials Science Forum, 2016, 836-837: 256-262.

[35] SANTHOSH T V, GOPIKA V, GHOSH A K, et al. An approach for reliability prediction of instrumentation and control cables by artificial neural networks and weibull theory for probabilistic safety assessment of NPPs[J]. Reliability Engineering and System Safety, 2018，170: 31-44.

[36] TIAN Z G, WONG L, SAFAEI N. A neural network approach for remaining useful life prediction utilizing both failure and suspension histories[J]. Mechanical Systems \& Signal Processing, 2010， 24(5): 1542-1555.

[37] BEZAZI A, PIERCE S G, WORDEN K, et al. Fatigue life prediction of sandwich composite materials under flexural tests using a Bayesian trained artificial neural network[J]. International Journal of Fatigue， 2007， 29(4): 738-747.
[38] PIERCE S G, WORDEN K, BEZAZI A. Uncertainty analysis of a neural network used for fatigue lifetime prediction[J]. Mechanical Systems \& Signal Processing, 2008, 22(6): 1395-1411.

[39] POWELL M J D. Radial basis functions for multivariable interpolation: A review[C]//Algorithms for Approximation, 1987: $143-167$.

[40] BORS A G. Introduction of the Radial Basis Function (RBF) Networks[C]//Online Symposium for Electronics Engineers, 1996: 393-408.

[41] LIU H, FAN M J, ZENG Q H, et al. RBF network based on artificial immune algorithm and application of predicting the residual life of injecting water pipeline[C]//Sixth International Conference on Natural Computation, 2010: 1305-1309.

[42] LI S Z, LI X Y, JIANG T M. A prediction method of life and reliability for CSALT using Grey RBF neural networks[C]//nternational Conference on Industrial Engineering and Engineering Management, 2009 : 699-703.

[43] 李志刚, 刘伯颖, 李玲玲, 等. 基于小波包变换及 RBF 神经网络的继电器寿命预测[J]. 电工技术学报, 2015, 30(14): 233-240.

LI Zhigang, LIU Boying, LI Lingling, et al. Life prediction of relay based on wavelet packet transform and RBF neural network[J]. Transactions of China Electrotechnical Society, 2015, 30(14): 233-240.

[44] CHEN X S, XIAO H, GUO Y J, et al. A multivariate grey RBF hybrid model for residual useful life prediction of industrial equipment based on state data[J]. International Journal of Wireless and Mobile Computing, 2016, 10(1): 90-96.

[45] HUANG G B, ZHU Q Y, SIEW C K. Extreme learning machine: A new learning scheme of feedforward neural networks[C]//IEEE International Joint Conference on Neural Networks, 2004: 985-990.

[46] 李磊. 基于极限学习机的风机机械传动部件剩余寿命 预测研究[D]. 秦皇岛: 燕山大学, 2015.

LI Lei. Residual life prediction for mechanical drive components of wind turbine based on extreme learning machine[D]. Qinhuangdao: Yanshan University, 2015.

[47] LIU F, LIU Y B, CHEN F L, et al. Residual life prediction for ball bearings based on joint approximate diagonalization of eigen matrices and extreme learning machine[J]. Proceedings of the Institution of Mechanical Engineers Part C: Journal of Mechanical Engineering Science, 2015, 231(9): 1-13. 
[48] LIU Y B, HE B, LIU F, et al. Remaining useful life prediction of rolling bearings using PSR, JADE, and extreme learning machine[J]. Mathematical Problems in Engineering, 2016, 2016: 1-13.

[49] 杜占龙, 李小民, 席雷平, 等. 多分类概率极限学习机 及其在剩余使用寿命预测中的应用[J]. 系统工程与电 子技术，2015，37(12): 2777-2784。

DU Zhanlong, LI Xiaomin, XI Leiping, et al. Multi-class probabilistic extreme learning machine and its application in remaining useful life prediction[J]. Systems Engineering and Electronics, 2015，37(12): 2777-2784.

[50] YANG Z, BARALDI P, ZIO E. A comparison between extreme learning machine and artificial neural network for remaining useful life prediction[C]//Prognostics and System Health Management Conference, 2017: 1-7.

[51] WANG X Y, HAN M. Online sequential extreme learning machine with kernels for nonstationary time series prediction[J]. Neurocomputing, 2014, 145(145): 90-97.

[52] 何群, 李否, 江国乾, 等. 基于 PCA 和多变量极限学 习机的轴承剩余寿命预测 [J]. 中国机械工程, 2014, 25(7): 984-989.

HE Qun, LI Lei, JIANG Guoqian, et al. Residual life predictions for bearings based on PCA and MELM[J]. China Mechanical Engineering, 2014, 25(7): 984-989.

[53] 吴婧睿. 基于 GSO-ELM 的锂离子电池剩余寿命间接预 测方法研究[D]. 大连：大连海事大学， 2017.

WU Jingrui. Research on indirect prediction of remaining life based on GSO-ELM for Lithium-ion battety[D]. Dalian: Dalian Maritime University, 2017.

[54] JOACHIMS T. Making large-scale SVM Learning Practical[C]//Technische Universität Dortmund , Sonderforschungsbereich 475: Komplexitätsreduktion in multivariaten Datenstrukturen, 1998: 499-526.

[55] CHERKASSKY V, MA Y. Practical selection of SVM parameters and noise estimation for SVM regression[J]. Neural Networks, 2004, 17(1): 113-126.

[56] ROCCO C M, MORENO J A. Fast Monte Carlo reliability evaluation using support vector machine[J]. Reliability Engineering \& System Safety, 2002， 76(3): 237-243.

[57] HONG W C, PAI P F. Predicting engine reliability by support vector machines[J]. International Journal of Advanced Manufacturing Technology, 2006, 28(1-2): 154-161.

[58] MIAO J Q, LI X G, YE J H. Predicting research of mechanical gyroscope life based on wavelet support vector[C]//First International Conference on Reliability
Systems Engineering, 2016: 1-5.

[59] NIETO P J G, GARCÍA-GONZALO E, LASHERAS F $S$, et al. Hybrid PSO-SVM-based method for forecasting of the remaining useful life for aircraft engines and evaluation of its reliability[J]. Reliability Engineering \& System Safety, 2015, 138: 219-231.

[60] MAIOR C B S, MOURA M D C, LINS I D, et al. Remaining useful life estimation by empirical mode decomposition and support vector machine[J]. IEEE Latin America Transactions, 2016, 14(11): 4603-4610.

[61] CHEN Z Z, CAO S C, MAO Z J. Remaining useful life estimation of aircraft engines using a modified similarity and supporting vector machine (SVM) approach[J]. Energies, 2017, 11(1): 1-14.

[62] TIPPING M E, SMOLA A. Sparse Bayesian learning and the relevance vector machine[J]. Journal of Machine Learning Research, 2001, 1(3): 211-244.

[63] MAIO F D, TSUI K L, ZIO E. Combining relevance vector machines and exponential regression for bearing residual life estimation[J]. Mechanical Systems \& Signal Processing, 2012, 31(8): 405-427.

[64] SONG Y C, LIU D T, HOU Y D, et al. Satellite lithium-ion battery remaining useful life estimation with an iterative updated RVM fused with the KF algorithm[J]. Chinese Journal of Aeronautics， 2018(1): 31-40.

[65] 王烨, 左洪福, 蔡景, 等. 基于 Bayesian 推断和 LS-SVM 的发动机在翼寿命预测模型 $[\mathrm{J}]$. 南京理工大学学报, 2013, 37(6): 955-959.

WANG Ye, ZUO Hongfu, CAI Jing, et al. Forecasting model of engine life on wing based on LS-SVM and Bayesian inference[J]. Journal of Nanjing University of Science and Technology，2013，37(6): 955-959.

[66] HINTON G E, OSINDERO S, TEH Y W. A fast learning algorithm for deep belief nets[J]. Neural Computation, 2006, 18(7): 1527-1554.

[67] SHAH S A A, BENNAMOUN M, BOUSSAID F. Iterative deep learning for image set based face and object recognition[J]. Neurocomputing, 2016, 174: 866-874.

[68] DENG L. Deep learning: From speech recognition to language and multimodal processing[J]. Apsipa Transactions on Signal \& Information Processing, 2016, 5: $1-15$.

[69] HE M, HE D. Deep learning based approach for bearing fault diagnosis[J]. IEEE Transactions on Industry Applications, 2017, 53(3): 3057-3065.

[70] REN L, CUI J, SUN Y Q, et al. Multi-bearing remaining useful life collaborative prediction: A deep learning 
approach[J]. Journal of Manufacturing Systems, 2017, 43: 248-256.

[71] ZHAO R, YAN R Q, CHEN Z H, et al. Deep learning and its applications to machine health monitoring: A survey[C]// IEEE Transactions on Neural Networks and Learning Systems, arXiv: 1612.07640, 2016.

[72] REN L, SUN Y, CUI J, et al. Bearing remaining useful life prediction based on deep autoencoder and deep neural networks[J]. Journal of Manufacturing Systems, 2018, 48: 71-77.

[73] 雷亚国, 贾峰, 周昕, 等. 基于深度学习理论的机械装 备大数据健康监测方法 $[\mathrm{J}]$. 机械工程学报, 2015, 51(21): 49-56.

LEI Yaguo, JIA Feng, Zhou Xin, et al. A deep learning-based method for machinery health monitoring with big data[J]. Journal of Mechanical Engineering, 2015, 51(21): 49-56.

[74] 周福娜, 高育林, 王佳瑜, 等. 基于深度学习的缓变故 障早期诊断及寿命预测 [J]. 山东大学学报, 2017, 47(5): 30-37.

ZHOU Funa, GAO Yulin, WANG Jiayu, et al. Early diagnosis and life prognosis for slowlyvarying fault based on deep learning[J]. Journal of Shandong University, 2017, 47(5): 30-37.

[75] 王佳瑜. 数据驱动的缓变微小故障早期诊断及剩余寿 命预测[D]. 开封: 河南大学, 2017.

WANG Jiayu. Data-driven early diagnosis and residual useful life prediction of slowly varying small fault[D]. Kaifeng: Henan University, 2017.

[76] YAN H, WAN J, ZHANG C, et al. Industrial big data analytics for prediction of remaining useful life based on deep learning[J]. IEEE Access， 2018， 6: 17190-17197.

[77] FISCHER A, IGEL C. Progress in pattern recognition, image analysis, computer vision, and applications[M]. Berlin: Springer-Verlag, 2012.

[78] 单晨晨. 基于深度置信网络的发动机状态监控研究[D]. 天津：中国民航大学， 2017.

SHAN Chenchen. Research on the engine condition monitoring based on deep belief net[D]. Tianjin: Civil Aviation University of China, 2017.

[79] DEUTSCH J, HE D. Using deep learning based approaches for bearing remaining useful life prediction[C]//Annual Conference of the Prognostics and Health Management Society, 2016: 1-7.

[80] DEUTSCH J, HE D. Using deep learning-based approach to predict remaining useful life of rotating components[J]. IEEE Transactions on Systems Man \& Cybernetics
Systems, 2017, 48(1): 11-20.

[81] DEUTSCH J, HE M, HE D. Remaining useful life prediction of hybrid ceramic bearings using an integrated deep learning and particle filter approach[J]. Applied Sciences, 2017, 649(7): 1-17.

[82] 张国辉. 基于深度置信网络的时间序列预测方法及其 应用研究[D]. 哈尔滨：哈尔滨工业大学, 2017.

ZHANG Guohui. Research on time series prediction and its application based on deep belief net work[D]. Harbin: Harbin Institute of Technology, 2017.

[83] ZHAO G Q，ZHANG G H, LIU Y F, et al. Lithium-ion battery remaining useful life prediction with deep belief network and relevance vector machine[C]//IEEE International Conference on Prognostics and Health Management, 2017: 7-13.

[84] ZHANG C, LIM P, QIN A K, et al. Multiobjective deep belief networks ensemble for remaining useful life estimation in prognostics[J]. IEEE Transactions on Neural Networks \& Learning Systems, 2016, 28(10): 2306-2318.

[85] LAWRENCE S, GILES C L, TSOI A C, et al. Face recognition: A convolutional neural-network approach[J]. IEEE Transactions on Neural Networks, 1997, 8(1): 98-113.

[86] SUN M L, SONG Z J, JIANG X H, et al. Learning pooling for convolutional neural network[J]. Neurocomputing, 2016, 224: 96-104.

[87] BABU G S, ZHAO P, LI X L. Database systems for advanced applications[M]. Berlin: Springer, 2016.

[88] LI X, DING Q, SUN J Q. Remaining useful life estimation in prognostics using deep convolution neural networks[J]. Reliability Engineering and System Safety, 2018, 172(C): 1-11.

[89] REN L, SUN Y, WANG H, et al. Prediction of bearing remaining useful life with deep convolution neural network[J]. IEEE Access， 2018， 6(99): 13041-13049.

[90] CHOW T W S, FANG Y. A recurrent neural-networkbased real-time learning control strategy applying to nonlinear systems with unknown dynamics[J]. IEEE Transactions on Industrial Electronics， 1998，45(1): 151-161.

[91] HEIMES F O. Recurrent neural networks for remaining useful life estimation[C]//International Conference on Prognostics and Health Management, 2008: 1-6.

[92] LIU J, SAXENA A, KAI G, et al. An adaptive recurrent neural network for remaining useful life prediction of lithium-ion batteries[C]//Annual Conference of the Prognostics and Health Management Society, 2010: 1-9.

[93] MALHI A, YAN R, GAO R X. Prognosis of defect 
propagation based on recurrent neural networks[J]. IEEE Transactions on Instrumentation \& Measurement, 2011, 60(3): 703-711.

[94] WU Y T, YUAN M, DONG S P, et al. Remaining useful life estimation of engineered systems using vanilla LSTM neural networks[J]. Neurocomputing, 2017, 275: 167-179.

[95] ZHANG Y Z, XIONG R, HE H W, et al. Long short-term memory recurrent neural network for remaining useful life prediction of lithium-ion batteries[J]. IEEE Transactions on Vehicular Technology, 2018, 67(7): 5695-5705.

[96] GUO L, LI N, JIA F, et al. A recurrent neural network based health indicator for remaining useful life prediction of bearings[J]. Neurocomputing, 2017, 240(C): 98-109.

[97] ZHANG J J, WANG P, YAN R Q, et al. Long short-term memory for machine remaining life prediction[J]. Journal of Manufacturing Systems, 2018, 48: 78-86.

作者简介: 裴洪, 男, 1992 年出生, 博士研究生。主要研究方向为装备 的剩余寿命预测与维修决策。

E-mail:ph2010hph@sina.com

胡昌华(通信作者), 男, 1966 年出生, 博士, 教授, 博士研究生导师, 长江学者特聘教授, 国家教学名师。主要研究方向为故障诊断、容错控 制、寿命预测与健康管理。

E-mail: hch_reu@sina.com 